\title{
Inclusion Relationships for Certain Subclasses of Meromorphic Functions Defined by Using the Extended Multiplier Transformations
}

\author{
R. M. El-Ashwah \\ Department of Mathematics, Faculty of Science (Damietta Branch), Mansoura University, \\ New Damietta 34517, Egypt \\ Correspondence should be addressed to R. M. El-Ashwah, elashwah@mans.edu.eg \\ Received 22 May 2011; Accepted 4 July 2011 \\ Academic Editors: A. Belhaj and A. Masiello \\ Copyright (C) 2012 R. M. El-Ashwah. This is an open access article distributed under the Creative \\ Commons Attribution License, which permits unrestricted use, distribution, and reproduction in \\ any medium, provided the original work is properly cited. \\ Let $\sum$ denote the class of analytic functions in the punctured unit disc $U^{*}=\{z: 0<|z|<1\}$. \\ Set $\varphi_{\lambda, \ell}^{m}(z)=1 / z+\sum_{k=0}^{\infty}[\ell+\lambda(k+1) / \ell]^{m} z^{k}\left(m \in \mathbb{N}_{0} ; \ell>0 ; \lambda \geq 0 ; z \in U^{*}\right)$, and define $\varphi_{\lambda, \ell}^{m, \mu}$ \\ in terms of the Hadamard product by $\varphi_{\lambda, \ell}^{m}(z) * \varphi_{\lambda, \ell}^{m, \mu}(z)=1 / z(1-z)^{\mu} \quad\left(\mu>0 ; z \in U^{*}\right)$. In this \\ paper, we introduce several new subclasses of analytic functions defined by means of the operator \\ $I_{\mu}^{m}(\lambda, \ell) f(z)=\varphi_{\lambda, \ell}^{m, \mu}(z) * f(z)\left(f \in \sum ; m \in \mathbb{N}_{0} ; \ell>0 ; \lambda \geq 0 ; \mu>0\right)$. Inclusion properties of these \\ classes and some applications involving integral operator are also considered.
}

\section{Introduction}

Let $\sum$ denote the class of functions of the form:

$$
f(z)=\frac{1}{z}+\sum_{k=0}^{\infty} a_{k} z^{k}
$$

which are analytic in the punctured open unit disk $U^{*}=\{z: z \in \mathbb{C}$ and $0<|z|<1\}=U \backslash\{0\}$. We denote by $\sum S(\eta), \sum K(\eta)$, and $\sum C(\eta, \beta)(0 \leq \eta, \beta<1)$ the subclasses of $\sum$ consisting of all meromorphic functions which are, respectively, starlike of order $\eta$ in $U$, convex of order $\eta$ in $U$, and close-to-convex of order $\eta$ and type $\beta$ in $U$ (see [1-3]).

Let $M$ be the class of all function $\varphi$ which are analytic and univalent in $U$ and for which $\varphi(U)$ is convex with

$$
\varphi(0)=1, \operatorname{Re}\{\varphi(z)\}>0 \quad(z \in U) .
$$


For two functions $f$ and $g$ analytic in $U$, we say that $f$ is subordinate to $g$ and write $f \prec g$ in $U$ or $f(z) \prec g(z)$, if there exists a Schwarz function $w(z)$, which is analytic in $U$ with $w(0)=0$ and $|w(z)|<1(z \in U)$, such that $f(z)=g(w(z))$. It is known that

$$
f(z) \prec g(z) \Longrightarrow f(0)=g(0), \quad f(U) \subset g(U)
$$

Furthermore, if the function $g$ is univalent in $U$ (see, [4, page 4]),

$$
f(z) \prec g(z) \Longleftrightarrow f(0)=g(0), \quad f(U) \subset g(U)
$$

Making use of the principle of subordination between analytic functions, we define the subclasses $\sum S(\eta ; \varphi), \sum K(\eta, \varphi)$, and $\sum C(\eta, \beta ; \varphi, \psi)$ of the class $\sum$ for $0 \leq \eta, \beta<1$ and $\varphi, \psi \in M$, which are defined by

$$
\begin{gathered}
\sum S(\eta, \varphi)=\left\{f: f \in \sum, \frac{1}{1-\eta}\left(\frac{-z f^{\prime}(z)}{f(z)}-\eta\right) \prec \varphi(z)(z \in U)\right\}, \\
\sum K(\eta, \varphi)=\left\{f: f \in \sum, \frac{1}{1-\eta}\left(-\left[1+\frac{z f^{\prime \prime}(z)}{f^{\prime}(z)}\right]-\eta\right) \prec \varphi(z)(z \in U)\right\}, \\
\sum C(\eta, \beta ; \varphi, \psi)=\left\{f: f \in \sum, \exists g \in S(\eta ; \varphi) \text { s.t. } \frac{1}{1-\beta}\left(-\frac{z f^{\prime}(z)}{g(z)}-\beta\right) \prec \varphi(z)(z \in U)\right\},
\end{gathered}
$$

respectively. For special choices for the parameters $\eta$ and $\beta$ as well as for special choices for the function $\varphi$ and $\psi$, we will obtain various subclasses of meromorphic function of the above classes (see [5-7]).

For $m \in \mathbb{N}_{0}=\mathbb{N} \cup\{0\}(\mathbb{N}=\{1,2, \ldots\})$, we define the multiplier transformation $J^{m}(\lambda, \ell)$ for functions $f \in \sum$ (see $[8,9]$ with $p=1$ ) by

$$
J^{m}(\lambda, \ell) f(z)=\frac{1}{z}+\sum_{k=0}^{\infty}\left(\frac{\ell+\lambda(k+1)}{\ell}\right)^{m} a_{k} z^{k} \quad\left(\ell>0 ; \lambda \geq 0 ; z \in U^{*}\right)
$$

Obviously, we have

$$
J^{m_{1}}(\lambda, \ell)\left(J^{m_{2}}(\lambda, \ell) f(z)\right)=J^{m_{2}}(\lambda, \ell)\left(J^{m_{1}}(\lambda, \ell) f(z)\right)=J^{m_{1}+m_{2}}(\lambda, \ell) f(z),
$$

for all integers $m_{1}$ and $m_{2}$.

We note that

(i) $J^{m}(1, \ell) f(z)=I(m, \ell) f(z)$ (see $\left.[10,11]\right)$;

(ii) $J^{m}(\lambda, 1) f(z)=D_{\lambda}^{m} f(z)$ (see [12]);

(iii) $J^{m}(1,1) f(z)=I^{m} f(z)$ (see [13]). 
Setting

$$
\varphi_{\lambda, \ell}^{m}(z)=\frac{1}{z}+\sum_{k=0}^{\infty}\left(\frac{\ell+\lambda(k+1)}{\ell}\right)^{m} z^{k} \quad\left(m \in \mathbb{N}_{0} ; \ell>0 ; \lambda \geq 0 ; z \in U^{*}\right),
$$

we define a new function $\varphi_{\lambda, \ell}^{m, \mu}(z)$ in terms of the Hadamard product (or convolution) by

$$
\varphi_{\lambda, \ell}^{m}(z) * \varphi_{\lambda, \ell}^{m, \mu}(z)=\frac{1}{z(1-z)^{\mu}} \quad\left(\mu>0 ; z \in U^{*}\right)
$$

Essentially Choi et al. [14] motivated the Choi-Saigo-Srivastava operator for analytic functions, which includes an integral operator considered earlier by Noor [15] and others [16-18]; we now introduce the operator $I_{\mu}^{m}(\lambda, \ell): \Sigma \rightarrow \sum$, which is defined here by;

$$
I_{\mu}^{m}(\lambda, \ell) f(z)=\varphi_{\lambda, \ell}^{m, \mu}(z) * f(z) \quad\left(f \in \sum ; m \in \mathbb{N}_{0} ; \ell>0 ; \lambda \geq 0 ; \mu>0\right) .
$$

We note that

(i) $I_{2}^{0}(1,1) f(z)=z f^{\prime}(z)+2 f(z)$ and $I_{2}^{1}(1,1) f(z)=f(z)$;

(ii) $I_{\mu}^{m}(1, \ell) f(z)=I_{\ell, \mu}^{m} f(z)$ (see [11]).

It is easily verified from the definition of the operator $I_{\mu}^{m}(\lambda, \ell)$ that

$$
\begin{gathered}
\lambda z\left(I_{\mu}^{m+1}(\lambda, \ell) f(z)\right)^{\prime}=\ell I_{\mu}^{m}(\lambda, \ell) f(z)-(\lambda+\ell) I_{\mu}^{m+1}(\lambda, \ell) f(z) \quad(\lambda>0), \\
z\left(I_{\mu}^{m}(\lambda, \ell) f(z)\right)^{\prime}=\mu I_{\mu+1}^{m}(\lambda, \ell) f(z)-(\mu+1) I_{\mu}^{m}(\lambda, \ell) f(z) .
\end{gathered}
$$

Next, by using the operator $I_{\mu}^{m}(\lambda, \ell)$ defined by (1.10), we introduce the following subclasses of meromorphic functions:

$$
\begin{array}{r}
\sum S_{\lambda, \ell}^{m, \mu}(\eta ; \varphi)=\left\{f: f \in \sum \text { and } I_{\mu}^{m}(\lambda, \ell) f(z) \in \sum S(\eta ; \varphi)\right\} \\
\left(\varphi \in M ; \lambda, \ell, \mu>0 ; m \in N_{0} ; 0 \leq \eta<1\right), \\
\sum K_{\lambda, \ell}^{m, \mu}(\eta ; \varphi)=\left\{f: f \in \sum \text { and } I_{\mu}^{m}(\lambda, \ell) f(z) \in \sum K(\eta ; \varphi)\right\} \\
\left(\varphi \in M ; \lambda, \ell, \mu>0 ; m \in N_{0} ; 0 \leq \eta<1\right), \\
\sum C_{\lambda, \ell}^{m, \mu}(\eta, \beta ; \varphi, \psi)=\left\{f: f \in \sum \text { and } I_{\mu}^{m}(\lambda, \ell) f(z) \in \sum C(\eta, \beta ; \varphi, \psi)\right\} \\
\quad\left(\varphi, \psi \in M ; \lambda, \ell, \mu>0 ; m \in N_{0} ; 0 \leq \eta, \beta<1\right) .
\end{array}
$$


We also note that

$$
f(z) \in \sum K_{\lambda, \ell}^{m, \mu}(\eta ; \varphi) \Longleftrightarrow-z f^{\prime}(z) \in \sum S_{\lambda, \ell}^{m, \mu}(\eta ; \varphi) .
$$

In particular, we set

$$
\begin{gathered}
\sum S_{\lambda, \ell}^{m, \mu}\left(\eta ; \frac{1+A z}{1+B z}\right)=\sum S_{\lambda, \ell}^{m, \mu}(\eta ; A, B) \quad(-1<B<A \leq 1), \\
\sum K_{\lambda, \ell}^{m, \mu}\left(\eta ; \frac{1+A z}{1+B z}\right)=\sum K_{\lambda, \ell}^{m, \mu}(\eta ; A, B) \quad(-1<B<A \leq 1) .
\end{gathered}
$$

The main object of this paper is to investigate several inclusion properties of the classes mentioned above. Some applications involving integral operator are also considered.

\section{Inclusion Properties Involving the Operator $I_{\mu}^{m}(\lambda, \ell)$}

The following lemmas will be required in our investigation.

Lemma 2.1 (see [19]). Let $\varphi$ be convex univalent in $U$ with $\varphi(0)=1$ and $\operatorname{Re}\{\beta \varphi(z)+\nu\}>0(\beta, v \in$ C). If $p$ is analytic in $U$ with $p(0)=1$, then

$$
p(z)+\frac{z p^{\prime}(z)}{\beta p(z)+v} \prec \varphi(z)
$$

implies that

$$
p(z) \prec \varphi(z)
$$

Lemma 2.2 (see [20]). Let $\varphi$ be convex univalent in $U$ and let $w$ be analytic in $U$ with $\operatorname{Re}\{w(z)\} \geq$ 0 . If $p(z)$ is analytic in $U$ and $p(0)=\varphi(0)$, then

$$
p(z)+w(z) z p^{\prime}(z) \prec p(z)
$$

implies that

$$
p(z) \prec \varphi(z)
$$

At first, with the help of Lemma 2.1, we prove the following theorem.

Theorem 2.3. Let $\varphi \in M$ with

$$
\max _{z \in U}(\operatorname{Re}\{\varphi(z)\})<\min \left(\frac{\mu+1-\eta}{1-\eta}, \frac{(\ell / \lambda)+1-\eta}{1-\eta}\right) \quad(\lambda, \mu, \ell>0 ; 0 \leq \eta<1)
$$


then

$$
\sum S_{\lambda, \ell}^{m, \mu+1}(\eta ; \varphi) \subset \sum S_{\lambda, \ell}^{m, \mu}(\eta ; \varphi) \subset \sum S_{\lambda, \ell}^{m+1, \mu}(\eta ; \varphi)
$$

Proof. We begin by showing the first inclusion relationship:

$$
\sum S_{\lambda, \ell}^{m, \mu+1}(\eta ; \varphi) \subset \sum S_{\lambda, \ell}^{m, \mu}(\eta ; \varphi)
$$

which is asserted by Theorem 2.3. Let $f \in \sum S_{\lambda, \ell}^{m, \mu+1}(\eta ; \varphi)$ and set

$$
p(z)=\frac{1}{1-\eta}\left(-\frac{z\left(I_{\mu}^{m}(\lambda, \ell) f(z)\right)^{\prime}}{I_{\mu}^{m}(\lambda, \ell) f(z)}-\eta\right)
$$

where the function $p(z)$ is analytic in $U$ with $p(0)=1$. Then, by applying (1.11) in (2.8), we obtain

$$
\mu \frac{I_{\mu+1}^{m}(\lambda, \ell) f(z)}{I_{\mu}^{m}(\lambda, \ell) f(z)}=-(1-\eta) p(z)+(\mu+1-\eta)
$$

Differentiating (2.9) logarithmically with respect to $z$ and multiplying the resulting equation by $z$, we have

$$
\frac{1}{1-\eta}\left(-\frac{z\left(I_{\mu+1}^{m}(\lambda, \ell) f(z)\right)^{\prime}}{I_{\mu+1}^{m}(\lambda, \ell) f(z)}-\eta\right)=\frac{z p^{\prime}(z)}{-(1-\eta) p(z)+\mu+1-\eta}+p(z) \quad(z \in U) .
$$

Since

$$
\max _{z \in U}(\operatorname{Re}\{\varphi(z)\})<\frac{\mu+1-\eta}{1-\eta} \quad(\mu>0 ; 0 \leq \eta<1 ; z \in U)
$$

we see that

$$
\operatorname{Re}\{\mu+1-\eta-(1-\eta) \varphi(z)\}>0 \quad(z \in U)
$$

Applying Lemma 2.1 to (2.10), it follows that $p \prec \varphi$ in $U$, that is,

$$
f \in \sum S_{\lambda, \ell}^{m, \mu}(\eta ; \varphi)
$$


For the second inclusion relationship asserted by Theorem 2.3, using arguments similar to those detailed above with (1.11), we obtain

$$
\sum S_{\lambda, \ell}^{m, \mu}(\eta, \beta ; \varphi) \subset \sum S_{\lambda, \ell}^{m+1, \mu}(\eta, \beta ; \varphi)
$$

We thus complete the proof of Theorem 2.3.

Theorem 2.4. Let $\varphi \in M$ with

$$
\max _{z \in U}(\operatorname{Re}\{\varphi(z)\})<\min \left(\frac{\mu+1-\eta}{1-\eta}, \frac{(\ell / \lambda)+1-\eta}{1-\eta}\right) \quad(\lambda, \mu, \ell>0 ; 0 \leq \eta<1)
$$

then

$$
\sum K_{\lambda, \ell}^{m, \mu+1}(\eta ; \varphi) \subset \sum K_{\lambda, \ell}^{m, \mu}(\eta ; \varphi) \subset \sum K_{\lambda, \ell}^{m+1, \mu}(\eta ; \varphi) .
$$

Proof. Applying (1.14) and Theorem 2.3, we observe that

$$
\begin{aligned}
& f(z) \in \sum K_{\lambda, \ell}^{m, \mu+1}(\eta ; \varphi) \Longleftrightarrow I_{\mu+1}^{m}(\lambda, \ell) f(z) \in \sum K(\eta ; \varphi) \\
& \Longleftrightarrow-z\left(I_{\mu+1}^{m}(\lambda, \ell) f(z)\right)^{\prime} \in \sum S(\eta ; \varphi) \\
& \Longleftrightarrow I_{\mu+1}^{m}(\lambda, \ell)\left(-z f^{\prime}(z)\right) \in \sum S(\eta ; \varphi) \\
& \Longleftrightarrow-z f^{\prime}(z) \in \sum S_{\lambda, \ell}^{m, \mu+1}(\eta ; \varphi) \\
& \Longrightarrow-z f^{\prime}(z) \in \sum S_{\lambda, \ell}^{m, \mu}(\eta ; \varphi) \\
& \Longleftrightarrow I_{\mu}^{m}(\lambda, \ell)\left(-z f^{\prime}(z)\right) \in \sum S(\eta ; \varphi) \\
& \Longleftrightarrow-z\left(I_{\mu}^{m}(\lambda, \ell) f(z)\right)^{\prime} \in \sum S(\eta ; \varphi) \\
& \Longleftrightarrow I_{\mu}^{m}(\lambda, \ell) f(z) \in \sum K(\eta ; \varphi) \\
& \Longleftrightarrow f(z) \in \sum K_{\lambda, \ell}^{m, \mu}(\eta ; \varphi),
\end{aligned}
$$




$$
\begin{aligned}
f(z) \in \sum K_{\lambda, \ell}^{m, \mu}(\eta ; \varphi) & \Longleftrightarrow-z f^{\prime}(z) \in \sum S_{\lambda, \ell}^{m, \mu}(\eta ; \varphi) \\
& \Longleftrightarrow-z f^{\prime}(z) \in S_{\lambda, \ell}^{m+1, \mu}(\eta ; \varphi) \\
& \Longleftrightarrow-z\left(I_{\mu}^{m+1}(\lambda, \ell) f(z)\right)^{\prime} \in \sum S(\eta ; \varphi) \\
& \Longleftrightarrow I_{\mu}^{m+1}(\lambda, \ell) f(z) \in \sum K(\eta ; \varphi) \\
& \Longleftrightarrow f(z) \in \sum K_{\lambda, \ell}^{m+1, \mu}(\eta ; \varphi)
\end{aligned}
$$

which evidently prove Theorem 2.4.

By setting

$$
\varphi(z)=\frac{1+A z}{1+B z} \quad(-1<B<A \leq 1 ; z \in U)
$$

in Theorems 2.3 and 2.4, we deduce the following corollary.

Corollary 2.5. Suppose that

$$
\frac{1+A}{1+B}<\min \left(\frac{\mu+1-\eta}{1-\eta}, \frac{(\ell / \lambda)+1-\eta}{1-\eta}\right) \quad(\lambda, \mu, \ell>0 ; 0 \leq \eta<1 ;-1<B<A \leq 1) .
$$

Then, for the function classes defined by (1.15),

$$
\begin{aligned}
& \sum S_{\lambda, \ell}^{m, \mu+1}(\eta ; A, B) \subset \sum S_{\lambda, \ell}^{m, \mu}(\eta ; A, B) \subset \sum S_{\lambda, \ell}^{m+1, \mu}(\eta ; A, B), \\
& \sum K_{\lambda, \ell}^{m, \mu+1}(\eta ; A, B) \subset \sum K_{\lambda, \ell}^{m, \mu}(\eta ; A, B) \subset \sum K_{\lambda, \ell}^{m+1, \mu}(\eta ; A, B) .
\end{aligned}
$$

Next by using Lemma 2.2, one obtains the following inclusion relationships for the class $\sum C_{\lambda, \ell}^{m, \mu}(\eta, \beta ; \varphi, \psi)$.

Theorem 2.6. Let $\varphi, \psi \in M$ with

$$
\max _{z \in U}(\operatorname{Re}\{\varphi(z)\})<\min \left(\frac{\mu+1-\eta}{1-\eta}, \frac{(\ell / \lambda)+1-\eta}{1-\eta}\right) \quad(\lambda, \mu, \ell>0 ; 0 \leq \eta<1) .
$$


Then

$$
\sum C_{\lambda, \ell}^{m, \mu+1}(\eta, \beta ; \varphi, \psi) \subset \sum C_{\lambda, \ell}^{m, \mu}(\eta, \beta ; \varphi, \psi) \subset \sum C_{\lambda, \ell}^{m+1, \mu}(\eta, \beta ; \varphi, \psi)
$$

Proof. We begin by proving that

$$
\sum C_{\lambda, \ell}^{m, \mu+1}(\eta, \beta ; \varphi, \psi) \subset \sum C_{\lambda, \ell}^{m, \mu}(\eta, \beta ; \varphi, \psi),
$$

which is the first inclusion relationship asserted by Theorem 2.6. Let

$$
f \in \sum C_{\lambda, \ell}^{m, \mu+1}(\eta, \beta ; \varphi, \psi)
$$

Then, in view of the definition of the function class $\sum C_{\lambda, \ell}^{m, \mu+1}(\eta, \beta ; \varphi, \psi)$, there exists a function $k(z) \in \sum S(\eta ; \varphi)$ such that

$$
\frac{1}{1-\beta}\left(-\frac{z\left(I_{\mu+1}^{m}(\lambda, \ell) f(z)\right)^{\prime}}{k(z)}-\beta\right) \prec \psi(z) .
$$

Choose the function $g(z)$ such that $I_{\mu+1}^{m}(\lambda, \ell) g(z)=k(z)$. Then $g(z) \in \sum S_{\lambda, \ell}^{m, \mu+1}(\eta ; \varphi)$ and

$$
\frac{1}{1-\beta}\left(-\frac{z\left(I_{\mu+1}^{m}(\lambda, \ell) f(z)\right)^{\prime}}{I_{\mu+1}^{m}(\lambda, \ell) g(z)}-\beta\right) \prec \psi(z) .
$$

Now let

$$
p(z)=\frac{1}{1-\beta}\left(-\frac{z\left(I_{\mu+1}^{m}(\lambda, \ell) f(z)\right)^{\prime}}{I_{\mu+1}^{m}(\lambda, \ell) g(z)}-\beta\right)
$$


where the function $p(z)$ is analytic in $U$ with $p(0)=1$. Using (1.12), we find that

$$
\begin{aligned}
\frac{1}{1-\beta}\left(-\frac{z\left(I_{\mu+1}^{m}(\lambda, \ell) f(z)\right)^{\prime}}{I_{\mu+1}^{m}(\lambda, \ell) g(z)}-\beta\right) \\
=\frac{1}{1-\beta}\left(\frac{I_{\mu+1}^{m}(\lambda, \ell)\left(-z f^{\prime}(z)\right)}{I_{\mu+1}^{m}(\lambda, \ell) g(z)}-\beta\right) \\
=\frac{1}{1-\beta}\left(\frac{z\left(I_{\mu}^{m}(\lambda, \ell)\left(-z f^{\prime}(z)\right)^{\prime}+(\mu+1) I_{\mu}^{m}(\lambda, \ell)\left(-z f^{\prime}(z)\right)\right.}{z\left(I_{\mu}^{m}(\lambda, \ell) g(z)\right)^{\prime}+(\mu+1) I_{\mu}^{m}(\lambda, \ell) g(z)}-\beta\right) \\
=\frac{1}{1-\beta} \\
\quad \times\left(\frac{\left(z\left(I_{\mu}^{m}(\lambda, \ell)\left(-z f^{\prime}(z)\right)^{\prime} / I_{\mu}^{m}(\lambda, \ell) g(z)\right)+(\mu+1)\left(I_{\mu}^{m}(\lambda, \ell)\left(-z f^{\prime}(z)\right) / I_{\mu}^{m}(\lambda, \ell) g(z)\right)\right.}{\left(z\left(I_{\mu+1}^{m}(\lambda, \ell) g(z)\right)^{\prime} / I_{\mu}^{m}(\lambda, \ell) g(z)\right)+(\mu+1)}-\beta\right) .
\end{aligned}
$$

Since

$$
g \in \sum S_{\lambda, \ell}^{m, \mu+1}(\eta ; \varphi) \subset \sum S_{\lambda, \ell}^{m, \mu}(\eta ; \varphi)
$$

by Theorem 2.3, then we set

$$
q(z)=\frac{1}{1-\eta}\left(-\frac{z\left(I_{\mu}^{m}(\lambda, \ell) g(z)\right)^{\prime}}{I_{\mu}^{m}(\lambda, \ell) g(z)}-\eta\right)
$$

where $q \prec \varphi$ in $U$ with the assumption that $\varphi \in M$. Then by (2.27) and (2.28), we observe that

$$
\begin{gathered}
I_{\mu}^{m}(\lambda, \ell)\left(-z f^{\prime}(z)\right)=(1-\beta) p(z) I_{\mu}^{m}(\lambda, \ell) g(z)+\beta I_{\mu}^{m}(\lambda, \ell) g(z) \\
\frac{1}{1-\beta}\left(-\frac{z\left(I_{\mu+1}^{m}(\lambda, \ell) f(z)\right)^{\prime}}{I_{\mu+1}^{m}(\lambda, \ell) g(z)}-\beta\right) \\
=\frac{1}{1-\beta}\left(\frac{\left(z\left(I_{\mu}^{m}(\lambda, \ell)\left(-z f^{\prime}(z)\right)^{\prime} / I_{\mu}^{m}(\lambda, \ell) g(z)\right)+(\mu+1)((1-\beta) p(z)+\beta)\right.}{\mu+1-\eta-(1-\eta) q(z)}-\beta\right)
\end{gathered}
$$


Differentiating both sides of (2.31) with respect to $z$, multiplying by $z$ and dividing by $I_{\mu}^{m}(\lambda, \ell) g(z)$, we obtain

$$
\frac{z\left(I_{\mu}^{m}(\lambda, \ell)\left(-z f^{\prime}(z)\right)^{\prime}\right.}{I_{\mu}^{m}(\lambda, \ell) g(z)}=(1-\beta) z p^{\prime}(z)-[(1-\beta) p(z)+\beta][(1-\eta) q(z)+\eta]
$$

Now making use of (2.26), (2.32), and (2.33), we get

$$
\frac{1}{1-\beta}\left(-\frac{z\left(I_{\mu}^{m}(\lambda, \ell) f(z)\right)^{\prime}}{I_{\mu}^{m}(\lambda, \ell) g(z)}-\beta\right)=p(z)+\frac{z p^{\prime}(z)}{(\mu+1-\eta)-(1-\eta) q(z)} \prec \psi(z)
$$

Since $\mu>0$ and $q \prec \varphi$ in $U$ with

$$
\max _{z \in U}(\operatorname{Re}\{\varphi(z)\})<\frac{\mu+1-\eta}{1-\eta}
$$

we have

$$
\operatorname{Re}\{\mu+1-\eta-(1-\eta) q(z)\}>0 \quad(z \in U)
$$

Hence, by taking

$$
w(z)=\frac{1}{(\mu+1-\eta)-(1-\eta) q(z)}
$$

in (2.34), and then applying Lemma 2.2, we can show that $p \prec \psi$ in $U$, so that

$$
f \in \sum C_{\lambda, \ell}^{m, \mu}(\eta, \beta ; \varphi, \psi)
$$

For the second inclusion relationship asserted by Theorem 2.6, using arguments similar to those detailed above with (1.11), we obtain

$$
\sum C_{\lambda, \ell}^{m, \mu}(\eta, \beta ; \varphi, \psi) \subset \sum C_{\lambda, \ell}^{m+1, \mu}(\eta, \beta ; \varphi, \psi)
$$

We thus complete the proof of Theorem 2.6. 


\section{Inclusion Properties Involving the Integral Operator $F_{c}$}

In this section, we consider the integral operator $F_{c}$ (see, [4, page 11]) defined by

$$
F_{c}(f)=F_{c}(f)(z)=\frac{c}{z^{c+1}} \int_{0}^{z} t^{c} f(t) d t \quad\left(f \in \sum ; c>0\right)
$$

From the definition (3.1), it is easily verified that

$$
z\left(I_{\mu}^{m}(\lambda, \ell) F_{c}(f)(z)\right)^{\prime}=c I_{\mu}^{m}(\lambda, \ell) f(z)-(c+1) I_{\mu}^{m}(\lambda, \ell) F_{c}(f)(z)
$$

By using (3.2) we can prove the following theorems (see Cho et al. [11]).

Theorem 3.1. Let $\varphi \in M$ with

$$
\max _{z \in U}(\operatorname{Re}\{\varphi(z)\})<\frac{c+1-\eta}{1-\eta} \quad(c>0 ; 0 \leq \eta<1) .
$$

If $f \in \sum S_{\lambda, \ell}^{m, \mu}(\eta ; \varphi)$, then

$$
F_{c}(f) \in \sum S_{\lambda, \ell}^{m, \mu}(\eta ; \varphi)
$$

Theorem 3.2. Let $\varphi \in M$ with

$$
\max _{z \in U}(\operatorname{Re}\{\varphi(z)\})<\frac{c+1-\eta}{1-\eta} \quad(c>0 ; 0 \leq \eta<1) .
$$

If $f \in \sum K_{\lambda, \ell}^{m, \mu}(\eta ; \varphi)$, then

$$
F_{c}(f) \in \sum K_{\lambda, \ell}^{m, \mu}(\eta ; \varphi)
$$

From Theorems 3.1 and 3.2, we can easily deduce the following.

Corollary 3.3. Suppose that

$$
\frac{1+A}{1+B}<\frac{c+1-\eta}{1-\eta} \quad(c>0 ;-1<B<A \leq 1 ; 0 \leq \eta<1) .
$$


Then for the function classes defined by (1.15), the following inclusion relationships hold true:

$$
\begin{gathered}
f \in \sum S_{\lambda, \ell}^{m, \mu}(\eta ; A, B) \Longrightarrow F_{c}(f) \in \sum S_{\lambda, \ell}^{m, \mu}(\eta ; A, B), \\
f \in \sum K_{\lambda, \ell}^{m, \mu}(\eta ; A, B) \Longrightarrow F_{c}(f) \in \sum K_{\lambda, \ell}^{m, \mu}(\eta ; A, B) .
\end{gathered}
$$

Theorem 3.4. Let $\varphi, \psi \in M$ with

$$
\max _{z \in U}(\operatorname{Re}\{\varphi(z)\})<\frac{c+1-\eta}{1-\eta} \quad(c>0 ; 0 \leq \eta<1) .
$$

If $f \in \sum C_{\lambda, \ell}^{m, \mu}(\eta, \beta ; \varphi, \psi)$, then

$$
F_{c}(f) \in \sum C_{\lambda, \ell}^{m, \mu}(\eta, \beta ; \varphi, \psi) .
$$

Remark 3.5. Putting $\lambda=1$ in all the above results, we will obtain the results obtained by Cho et al. [11].

\section{Acknowledgment}

The author thanks the referees for their valuable suggestions which led to improvement of this paper.

\section{References}

[1] V. Kumar and S. L. Shukla, "Certain integrals for classes of $p$-valent meromorphic functions," Bulletin of the Australian Mathematical Society, vol. 25, no. 1, pp. 85-97, 1982.

[2] R. J. Libera and M. S. Robertson, "Meromorphic close-to-convex functions," The Michigan Mathematical Journal, vol. 8, pp. 167-176, 1961.

[3] H. M. Srivastava and S. Owa, Current Topics in Analytic Function Theory, World Scientific Publishing, Hackensack, NJ, USA, 1992.

[4] S. S. Miller and P. T. Mocanu, Differential Subordinations: Theory and Application, vol. 225 of Monographs and Textbooks in Pure and Applied Mathematics, Marcel Dekker, New York, NY, USA, 2000.

[5] S. K. Bajpai, "A note on a class of meromorphic univalent functions," Revue Roumaine de Mathématiques Pures et Appliquées, vol. 22, no. 3, pp. 295-297, 1977.

[6] R. M. Goel and N. S. Sohi, "On a class of meromorphic functions," Glasnik Matematički III, vol. 17, no. 37, pp. 19-28, 1982.

[7] R. Singh, "Meromorphic close-to-convex functions," Journal of the Indian Mathematical Society, vol. 33, pp. 13-20, 1969.

[8] R. M. El-Ashwah, "A note on certain meromorphic p-valent functions," Applied Mathematics Letters, vol. 22, no. 11, pp. 1756-1759, 2009.

[9] R. M. EL-Ashwah, M. K. Aouf, and T. Bulboaca, "Differential subordinations for classes of meromorphic $p$-valent functions defined by multiplier transformations," Bulletin of the Australian Mathematical Society, vol. 83, pp. 353-368, 2011.

[10] N. E. Cho, O. S. Kwon, and H. M. Srivastava, "Inclusion and argument properties for certain subclasses of meromorphic functions associated with a family of multiplier transformations," Journal of Mathematical Analysis and Applications, vol. 300, no. 2, pp. 505-520, 2004. 
[11] N. E. Cho, O. S. Kwon, and H. M. Srivastava, "Inclusion relationships for certain subclasses of meromorphic functions associated with a family of multiplier transformations," Integral Transforms and Special Functions, vol. 16, no. 8, pp. 647-659, 2005.

[12] F. M. Al-Oboudi and H. A. Al-Zkeri, "Applications of Briot-Bouquet differential subordination to some classes of meromorphic functions," Arab Journal of Mathematical Sciences, vol. 12, no. 1, pp. 1730, 2006.

[13] B. A. Uralegaddi and C. Somanatha, "New criteria for meromorphic starlike univalent functions," Bulletin of the Australian Mathematical Society, vol. 43, no. 1, pp. 137-140, 1991.

[14] J. H. Choi, M. Saigo, and H. M. Srivastava, "Some inclusion properties of a certain family of integral operators," Journal of Mathematical Analysis and Applications, vol. 276, no. 1, pp. 432-445, 2002.

[15] K. I. Noor, "On new classes of integral operators," Journal of Natural Geometry, vol. 16, no. 1-2, pp. 71-80, 1999.

[16] J.-L. Liu, "The Noor integral and strongly starlike functions," Journal of Mathematical Analysis and Applications, vol. 261, no. 2, pp. 441-447, 2001.

[17] J.-L. Liu and K. I. Noor, "Some properties of Noor integral operator," Journal of Natural Geometry, vol. 21, no. 1-2, pp. 81-90, 2002.

[18] K. I. Noor and M. A. I. Noor, “On Integral Operators," Journal of Mathematical Analysis and Applications, vol. 238, no. 2, pp. 341-352, 2001.

[19] P. Eenigenburg, S. S. Miller, P. T. Mocanu, and M. O. Reade, “On a Briot-Bouquet differential subordination," in General Inequalities 3, vol. 64, pp. 339-348, Birkhäuser, Basel, Switzerland, 1983.

[20] S. S. Miller and P. T. Mocanu, "Differential subordinations and univalent functions," The Michigan Mathematical Journal, vol. 28, no. 2, pp. 157-172, 1981. 


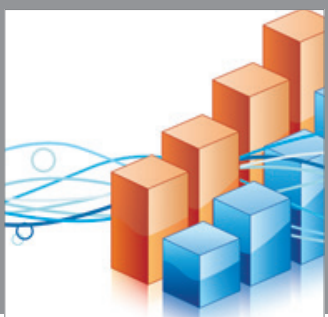

Advances in

Operations Research

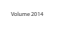

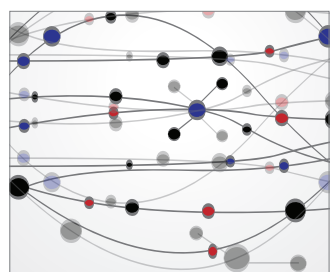

\section{The Scientific} World Journal
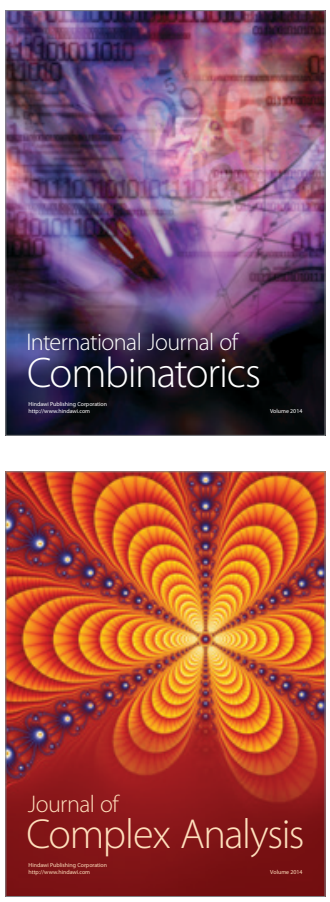

International Journal of

Mathematics and

Mathematical

Sciences
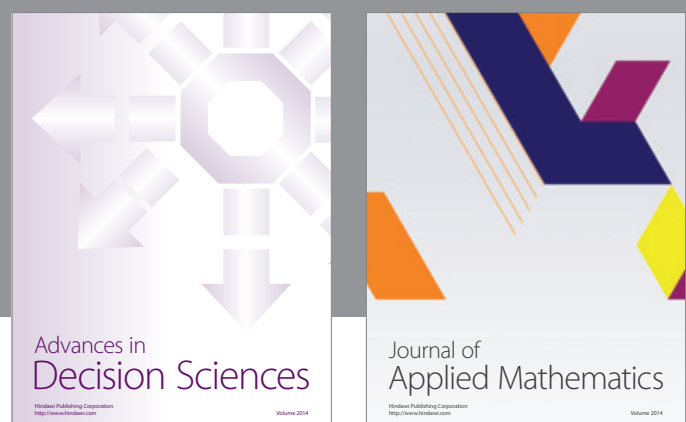

Journal of

Applied Mathematics
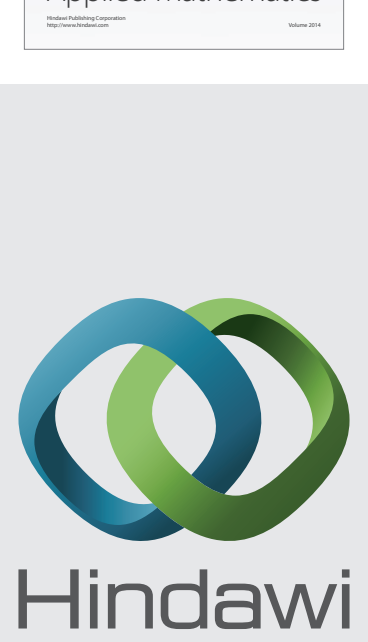

Submit your manuscripts at http://www.hindawi.com
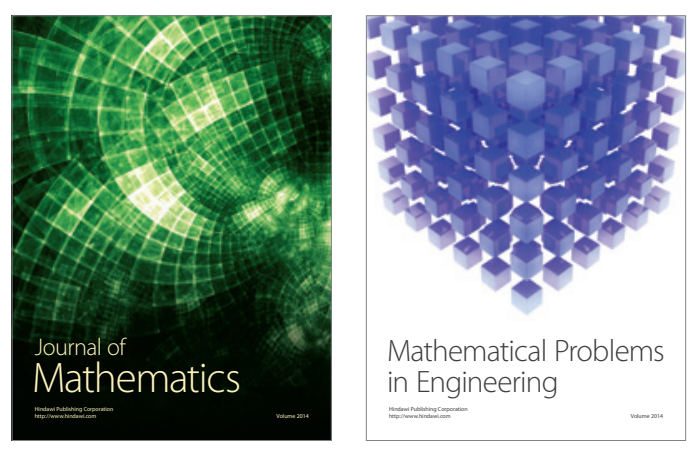

Mathematical Problems in Engineering
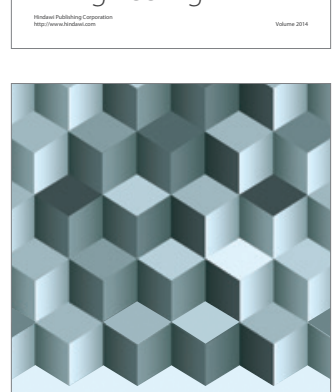

Journal of

Function Spaces
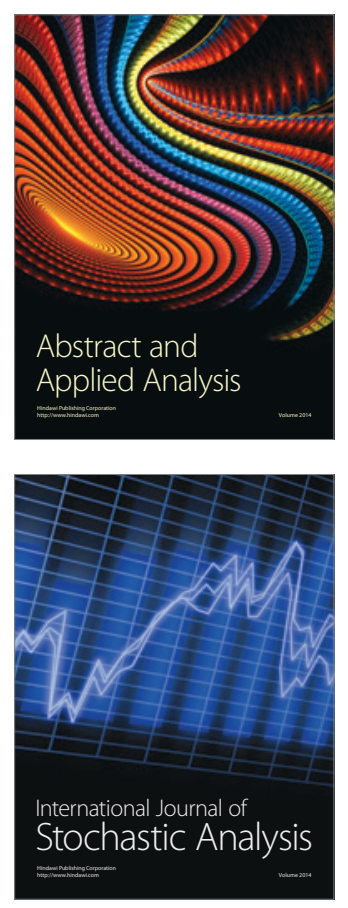

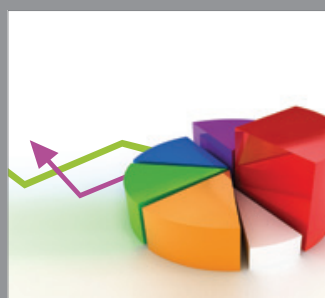

ournal of

Probability and Statistics

Promensencen
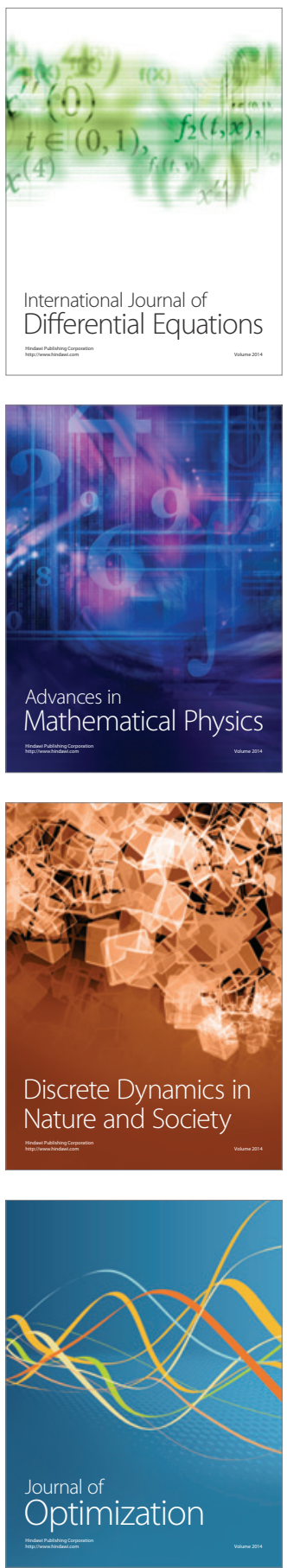